\title{
Onboard Processor for Compressing HSI Data
}

\author{
Sid Cook \\ Lockheed Martin Space Systems - Astronautics, Denver CO \\ Joseph Harsanyi \\ Applied Signal \& Image Technology, Glen Burnie, MD
}

\begin{abstract}
With EO-1 Hyperion and MightySat in orbit NASA and the DoD are showing their continued commitment to hyperspectral imaging (HSI). As HSI sensor technology continues to mature, the ever-increasing amounts of sensor data generated will result in a need for more cost effective communication and data handling systems. Lockheed Martin, with considerable experience in spacecraft design and developing special purpose onboard processors, has teamed with Applied Signal \& Image Technology (ASIT), who has an extensive heritage in HSI, to develop a real-time and intelligent onboard processing (OBP) system to reduce HSI sensor downlink requirements. Our goal is to reduce the downlink requirement by a factor $>100$, while retaining the necessary spectral fidelity of the sensor data needed to satisfy the many science, military, and intelligence goals of these systems. Our initial spectral compression experiments leverage commercial-off-the-shelf (COTS) spectral exploitation algorithms for segmentation, material identification and spectral compression that ASIT has developed. ASIT will also support the modification and integration of this COTS software into the OBP. Other commercially available COTS software for spatial compression will also be employed as part of the overall compression processing sequence.

Over the next year elements of a high-performance reconfigurable OBP will be developed to implement proven preprocessing steps that distill the HSI data stream in both spectral and spatial dimensions. The system will intelligently reduce the volume of data that must be stored, transmitted to the ground, and processed while minimizing the loss of information.
\end{abstract}

\section{INTRODUCTION}

Our objective is to reduce spacecraft cost and increase the accessibility and utility of HSI data through appropriate use of onboard processing. Our strategy is to combine the processing expertise from ASIT, a leader in the HSI processing field and the developer of a library of HSI directed data compression and target detection algorithms, with the LM remote sensing, spacecraft and OBP capabilities. During the first eighteen months we are identifying, modifying and testing ASIT-developed algorithms as candidates for the OBP. We will then leverage the technologies available through LM to develop an OBP architecture concept that would support the throughput, flexibility and fidelity required by the sensor and the scientific community that use the data. Our heterogeneous OBP capabilities combine large processing throughput with a high degree of fidelity and reprogrammability by integrating state-of-the-art digital signal processors (DSPs) and field programmable gate arrays (FPGAs). ASIC and optical processing using high density interconnect (HDI) technology are available for future applications. This innovative combination of processing and packaging technologies will enable this processor to be used onboard the satellite as well as in analyst or mobile ground workstations.

Our technical effort began with studies to identify the algorithm functions that provide the best value as OBP processes. We will partition the processing requirements to the different elements of our initial DSP and FPGA processing architecture, develop both software and hardware-in-the-loop simulations for certain elements, and generate roadmaps for advancing the OBP to flight status.

\section{SYSTEM CONCEPT}

Figure 1 presents the HSI processing chain from sensor to finished HSI output products. Our OBP compression algorithms are designed to run unsupervised using only the data statistics to determine the compression transformation. The calibrated sensor data are input to our directed data compression processing flow and compressed.

The compressed data are then transmitted to the ground where they are uncompressed, corrected to account for sensor calibration results thus converting raw to radiance data, and atmospheric absorption effects are removed from the spectra thus resulting in reflectance data. Material classification and identification can then be performed using standard techniques. This information can then be used to generate various image-based products; e.g., classification and abundance maps. 


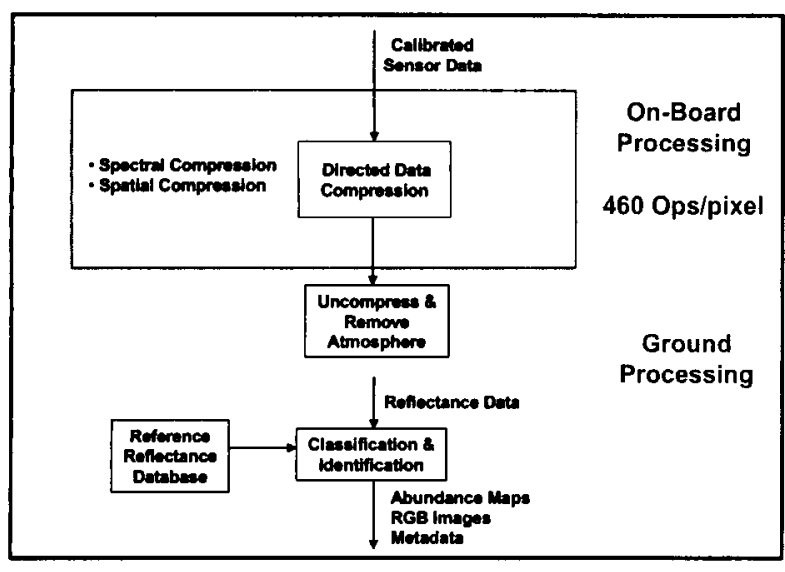

Figure 1 HSI Data Processing Chain

A top-level description of our directed data compression is presented in Figure 2 . In step 1 dominant pixels are separated from anomalous pixels using the tunable anomaly detector as shown in Figure 3. This initial segmentation is important since dominant and anomalous pixels will be compressed differently; hence the name "directed data compression". This separation occurs by first determining the second order statistics and estimating the number of eigenvectors associated with the dominant pixels. The pixels that do not belong to the dominant subspace are automatically thresholded and so those pixels that have inherently large amounts of information content are labeled anomalous. The anomalous pixels are passed along to the Spectral Uniqueness Monitor (SUM) where the subset of spectral signatures required to describe all anomalous pixel are determined. These spectral signatures are the anomalous subspace endmembers. The automatic subspace discrimination function is then used to assign each anomalous pixel to one of the SUM derived classes.

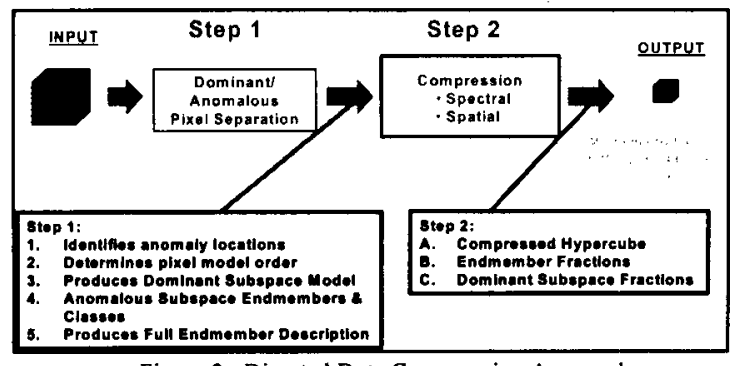

Figure 2 Directed Data Compression Approach

For Step 2, in Figure 2, the anomalous spectral basis signatures along with the dominant subspace eigenvectors are used together to formulate a compressing transformation in the spectral dimension, as shown in Figure 3. A COTS spatial compression algorithm is then applied to further increase the overall compression ratio. Currently, the most promising algorithm uses wavelet basis functions to provide the compression. Preliminary results show that an additional $10 \mathrm{X}$ compression can be achieved spatially before degrading the data to the extent that science information is dramatically impacted. Thus with $10 \mathrm{X}$ compression for both spectrally and spatially a 100:1 compression results.

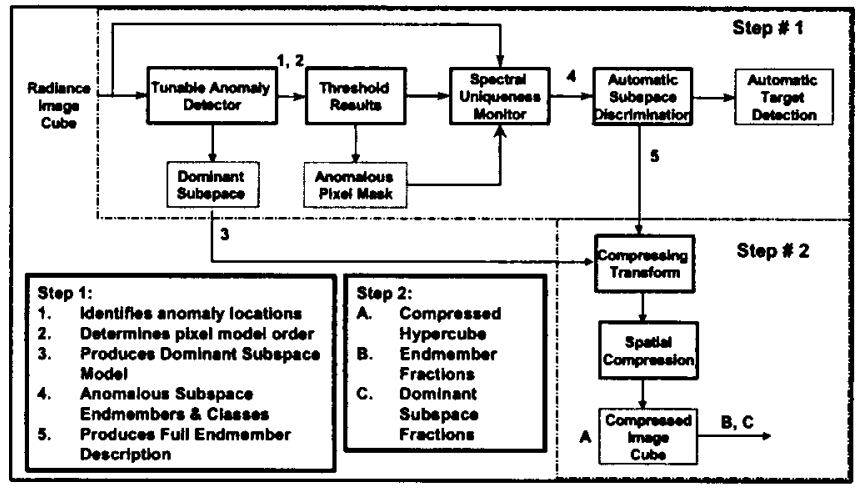

Figure 3 Directed Data Compression Processing Flow

\section{EXAMPLE OF HSI DIRECTED DATA COMPRESSION RESULTS}

Figures 4 and 5 present the results of an approximate 100:1 compression of the Cuprite and Coleamberally scenes acquired from the EO- 1 satellite using the 242 band Hyperion sensor. Since 44 bands of the satellite did not function properly only 198 bands were calibrated and used for analysis. Even though little or no visual differences can be observed in Figure 4 or 5 there are some spectral differences. These spectral differences can be observed by comparing the spectral angles (angles, measured in degrees, determined by computing the spectral dot product) for corresponding pixels in the original and compressed data cubes. Small angles imply small differences in spectral data between the original and compressed data cubes. These computations were performed for every pixel in the scenes as observed in Figures 4 and 5. 


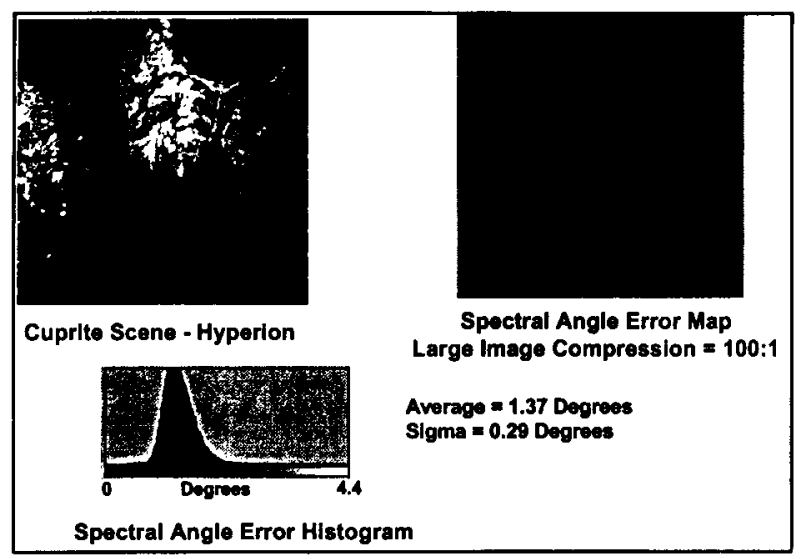

Figure 4 Spectral Angle Error-198 Calibrated Bands

In addition to spectral angle comparisons, it is also possible to compare individual spectral features of compressed and uncompressed signatures. This type of comparison is being done in collaboration with EO-1 project scientists to ensure high value science information is not lost in the compression process.

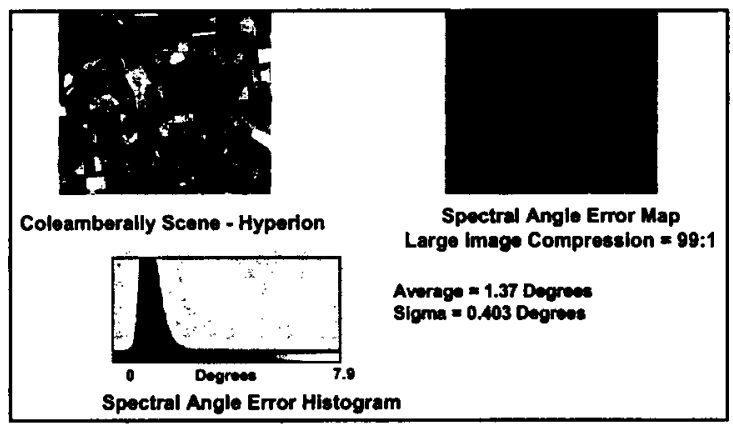

Figure 5 Spectral Angle Error - 198 Bands

Comparing Figures 4 and 5 one can observe that compression characteristics are scene dependent. In Figure 6, showing plots of the average spectral angle error and the standard deviation of that error versus compression ratios, the differences in the standard deviation curves between the scenes can be observed. The compression ratio does not take into account the size of the auxiliary data file because this file size is almost independent of the size of the data cube. Therefore for most operational strip maps the size of this auxiliary data file will be negligible in comparison to the compressed data cube.

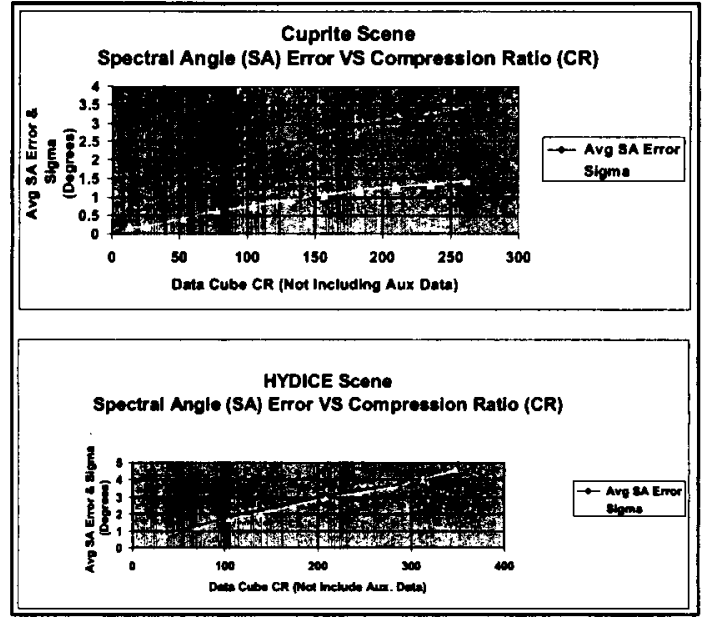

Figure 6 Spectral Angle Error Sensitivity

\section{ONBOARD PROCESSOR (OBP) REQUIREMENTS}

Approximately 460 floating-point operations per pixel of input sensor data are required to implement the directed data compression processing flow specified in Figure 3. This information combined with specific sensor characteristics results in the OBP data processing requirements summarized in Figure 7.

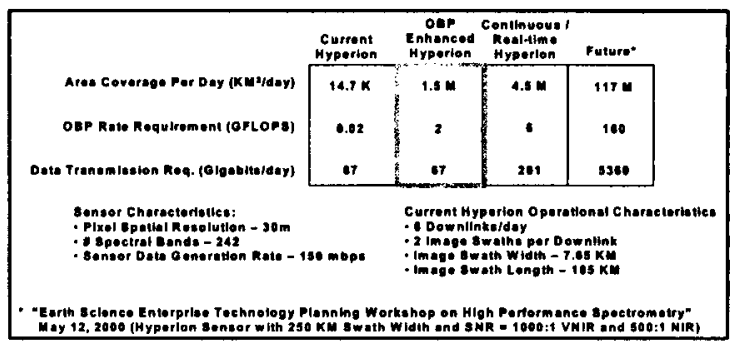

Figure 7 Onboard Processor Requirements

For the EO-1 Hyperion sensor under the current operational environment the minimum requirement for an OBP to keep up with the data on a daily basis is 0.02 GFLOPS of equivalent computational power. Since our goal is to compress the data by a factor of 100:1 a Hyperion sensor could theoretically be tasked to increase its coverage by a factor of 100 without increasing the downlink requirements with the OBP processing capability of 2 GFLOPS equivalent, which we have chosen as the baseline requirements for the OBP system we plan to develop. If the Hyperion sensor is operated continuously then the OBP requirement becomes 6 GFLOPS equivalent. NASA Scientists at the May 2000 meeting of "Earth Science Enterprise Technology Planning Workshop on High Performance Spectrometry" specified a "future" HSI sensor with 
similar sensor characteristics to the Hyperion except with much improved noise characteristics and a swath increase from $7.5 \mathrm{~km}$ to $250 \mathrm{~km}$. The corresponding OBP requirement to operate this future sensor continuously would be 160 GFLOPS. Analysis of the Directed Data Compression algorithms to be implemented by the OBP shows the single most computational intensive operation to be the matrix multiply operation. For a Hyperion type sensor this means operation with 256 pixel X 242 band sensor data matrices. A single generic matrix multiply operation (MMOp) on such data corresponds to approximately 16 million multiplies and adds, when reduced to 16-bit integer arithmetic. A 2 GFLOP equivalent OBP would be capable of performing approximately 120 such MMOps/sec. For comparison, a desktop computer equipped with a $1.2 \mathrm{GHz}$ Pentium $^{\mathrm{TM}}$ processor can perform $10 \mathrm{MMOps} / \mathrm{sec}$. Our proposed OBP breadboard design for the Nov 2002 demo using 50 $\mathrm{MHz}$ FPGA based matrix multiply processing engines should be able to achieve at least $30 \mathrm{MMOps} / \mathrm{sec}$.

\section{Proposed OBP Architecture}

In developing our proposed OBP architecture, several key points have been used as a guiding philosophy. First of all, the architecture should be modular in nature so as to be readily adaptable to future processing needs, both from a sensor type and overall throughput standpoint. A high degree of modularity also allows the maximization of parallel processing paradigms. Second, the concept should be highly FPGA based so as to maximize programmability and reconfigurability to accommodate changing mission computational requirements. Third, the use of COTS standards should be used where feasible to minimize development costs, however component selections should be done with caution so as to not preclude a clear path to a radtolerant implementations. Figure 8 illustrates such OBP architecture.

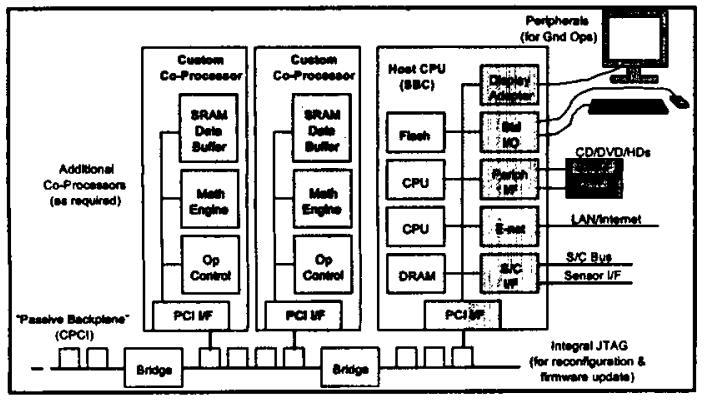

Figure 8 System Architecture
Our concept is built around an industry standard Compact PCI (CPCI) backplane, which offers appropriate robustness for spaceborn applications, in addition to expandability, and data bandwidth. Modular FPGA and/or DSP based co-processor modules can be implemented as future processing needs expand. The "host" system processor serves as an overall OBP housekeeping controller, provides standard external user interfaces for checkout and manufacturing, and can also share in the OBP data compression tasks, although most of the computational intensive operations will be distributed to appropriate co-processor modules. A choice of standard processors is available for the host function, such as the currently prevalent Power PCTM processor products. Real-time embedded operating systems such as VXWorks or LynxOS would be an appropriate choice for an autonomous spaceborn OBP. The CPCI backplane protocol readily supports independent "bus mastering" by any of the co-processor modules to implement direct memory access (DMA) data transfers between modules. As OBP processing demands exceed the inherent capacity of the CPCI backplane, direct high-speed point-to-point data interfaces can be implemented between appropriate coprocessor modules.

Perhaps more importantly, the basic architecture permits an easily implemented incremental development and demonstration roadmap, which can leverage conventional desktop PC technology, Windows development environments, and other COTS products prior to eventual migration to a fully flight qualified OBP.

As an initial step in such an incremental development plan, Figure 9 depicts the functionality to be implemented in preparation for a November 2002 breadboard demonstration. This single co-processor card will be FPGA based, and designed using Xilinx XCV1000 and XCV300 components that are currently available in a rad-tolerant version, so as not to preclude eventual design migration to an OBP. The PCI backplane interface, external data port control, and local data bus transfers will all be implemented in FPGA logic to demonstrate the independent bus mastering, multiprocessor architecture operating in a Windows 2000 environment. The matrix multiply math function, along with necessary data sequencing, row and column data buffering, and overall instruction execution logic will also be implemented in FPGA logic to demonstrate the performance advantages possible from dedicated processing hardware. An ample amount of high-speed SDRAM will provide local storage of matrix data necessary to support any given calculation. 


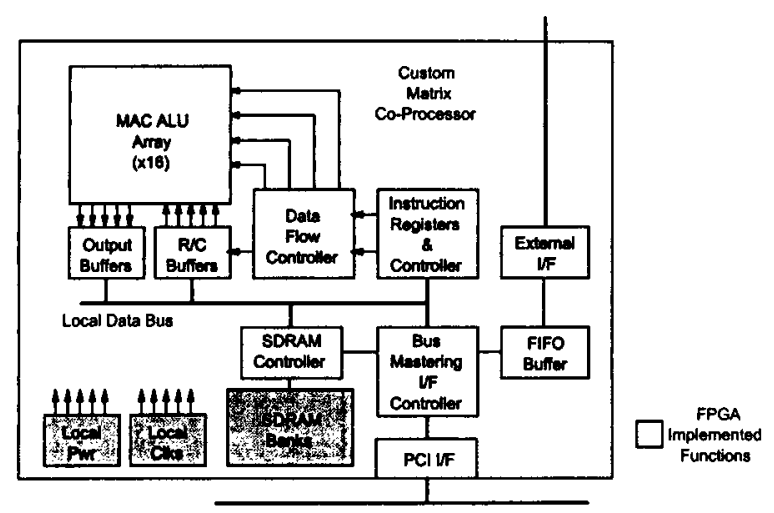

Figure 9 Matrix Multiply Co-Processor

The matrix multiply co-processor card will receive its instructions from the host, which will consist of an "opcode" which defines the exact operation to be performed, the size of the data arrays, and pointers to the data locations in host memory. The co-processor card will then initiate DMA operations to retrieve the operand data, execute the matrix multiply instruction, return the results to host memory, and generate an interrupt to the host processor signifying instruction completion. Although initially implemented in a conventional PC, this approach serves to demonstrate the advantages of parallel co-processors within the overall OBP architecture, and does not preclude a simple migration path to a real-time host operating system and fully flight qualified hardware in the future.

\section{CONCLUSIONS}

We have now defined and demonstrated an approach to compress HSI data, and have shown that we can compress these data 100:1 without obvious visual and spectral degradation. When we compare the spectra of corresponding pixels between compressed and uncompressed data cubes we observe angular differences and other slight changes in spectral features. The significance of these differences is currently unknown. Currently NASA scientists are supporting us in evaluating the significance of the differences between the compressed and uncompressed data cubes, so that important science data are not lost.

We are currently recommending a design requirement for the OBP of 2 GFLOPS for this contract, as indicated by the highlighted box in Figure 7 . We believe that digital signal processor (DSP) and field programmable gate array (FPGA) technology applied in a modular parallel processing architecture as shown in Figure 8 can satisfy both near-term and long-term flight-worthy OBP requirements. Other enabling technologies; e.g., application-specific integrated circuits (ASIC), optical processors, and high-density interconnect (HDI) packaging, are available to address future OBP requirements as volume and power constraints become more prohibitive.

\section{ACKNOWLEDGMENTS}

This work is being performed under the NASA/ESTO/AIST NRA contract NAS5-00216.

\section{REFERENCES}

Harsanyi, J. C., (1993), Detection and Classification of Subpixel Spectral Signatures in Hyperspectral Image Sequences, Ph.D. Dissertation, University of Maryland, Baltimore County, $116 \mathrm{pp}$.

Harsanyi, J. C. and C. Chang, "Hyperspectral Image Classification and Dimensionality Reduction: An Orthogonal Subspace Projection Approach," IEEE Transactions on Geoscience and Remote Sensing, July, 1994.

Harsanyi, J. C. and C. Chang, " Hyperspectral Image Dimensionality Reduction and Pixel Classification: An Orthogonal Subspace Projection Approach," Proceedings of the Twenty-seventh Annual Conference on Information Sciences and Systems, pp. 401-406, Mar 24-26, 1993.

Harsanyi, J. C., W. H. Farrand, C. Chang, "Detection of Subpixel Signatures in Hyperspectral Image Sequences," 1994 ASPRS/ACSM Annual Convention and Exposition Technical Papers, Reno, Nevada, vol. 1, pp. 236-247, Apr. 25 -28, 1994. 